\title{
Invasive Pulmonary Aspergillosis and Nocardiosis in a Child with Possible Hyperimmunoglobulin E Syndrome
}

\author{
Savita G Krishnamurthy ${ }^{1}$, Chikkanayakanahalli Indumathi ${ }^{2}$, Kanchamaranahalli L Madhura $^{3}$, Raghavendra Kulkarni ${ }^{4}$
}

\begin{abstract}
Aim and objective: To highlight the importance of looking for rare opportunistic as well as coinfections in children with primary immunodeficiency. Background: Opportunistic infections are often seen in children with primary immunodeficiency. Infections like nocardiosis and aspergillosis often mimic tuberculosis. So far, a single case of nocardiosis in hyperimmunoglobulin E syndrome (HIES) has been reported but none with coinfection with aspergillosis. Here we report a child with possible HIES presenting with nocardiosis and invasive pulmonary aspergillosis.

Case description: A 3-year-old boy with recurrent abscesses and pneumonia presented to us with persistent fever and cough for 5 months, unresponsive to antitubercular therapy. Considering the suggestive history, characteristic facies, and elevated immunoglobulin E levels, HIES was diagnosed based on the NIH (National Institute of Health)-HIES score. High-resolution CT chest revealed mediastinal lymphadenopathy with right-sided nodular infiltrates and bilateral bronchiectasis. A positive serum galactomannan assay and growth of Aspergillus in bronchoalveolar lavage confirmed invasive aspergillosis. Mediastinal lymph node aspirate showed acid fast bacilli on smear and culture grew Nocardia species. Conclusion: It is important to look for rare opportunistic as well as coinfections in children with primary immunodeficiency. To the best of our knowledge, ours is the first report of pulmonary aspergillosis and nocardiosis in a child with HIES.

Clinical significance: Albeit much rarer, aspergillosis and pulmonary nocardiosis can be close mimics of tuberculosis. Nonresolving pulmonary symptoms in the immunocompromised host must raise a suspicion of opportunistic infections including nocardiosis as early diagnosis and treatment may alter outcomes.
\end{abstract}

Keywords: Hyperimmunoglobulin E syndrome, Invasive aspergillosis, Nocardia, Primary Immunodeficiency, Tuberculosis.

Pediatric Infectious Disease (2021): 10.5005/jp-journals-10081-1288

\section{BACKGROUND}

Infection with uncommon organisms is the norm in children with primary and secondary immunodeficiencies. As tuberculosis (TB) is still the most common infection in an immunocompromised host, any pulmonary involvement is often treated with antitubercular therapy (ATT). However, infections with other opportunistic organisms like Nocardia and Aspergillus species go untreated as they closely mimic tuberculosis. Although colonization by Aspergillus is common in children with primary immunodeficiency, invasive aspergillosis with nocardiosis coinfection has rarely been reported in this population. So far, a single case of nocardiosis in hyperimmunoglobulin $\mathrm{E}$ syndrome (HIES) has been reported ${ }^{1}$ but none with coinfection with aspergillosis. Here we report a child with possible HIES presenting with nocardiosis and invasive aspergillosis.

\section{Case Description}

A 3-year-old boy, second born of a third-degree consanguineous marriage, to an apparently healthy couple, presented to us with persisting fever and cough since the last 5 months associated with poor weight gain and anorexia. He was already on ATT as his initial gastric aspirate done elsewhere was positive for acid fast bacilli (AFB), though culture did not show growth of Mycobacterium tuberculosis. Mycobacterial PCR by cartridge based nucleic acid amplification test (CBNAAT) was negative. In view of poor response to ATT, he had undergone a mediastinal lymph node aspirate before referral, which was positive for AFB. He had a history of recurrent pneumonia and skin abscesses since 6 months of age, including right testicular abscess requiring orchidectomy at \begin{tabular}{l}
\hline${ }^{1-3}$ Department of Pediatrics, St John's Medical College, Bengaluru, \\
Karnataka, India \\
${ }^{4}$ Department of Microbiology, SDM College of Medical Sciences and \\
Hospital, Dharwad, Karnataka, India \\
Corresponding Author: Savita G Krishnamurthy, Department of \\
Pediatrics, St John's Medical College, Bengaluru, Karnataka, India, \\
Phone: +917760778441 , e-mail: sivakripa@gmail.com \\
How to cite this article: Krishnamurthy SG, Indumathi C, Madhura \\
KL, et al. Invasive Pulmonary Aspergillosis and Nocardiosis in a Child \\
with Possible Hyperimmunoglobulin E Syndrome. Pediatr Inf Dis
\end{tabular} with Possible Hyp

Source of support: Nil

Conflict of interest: None

1 year of age. There was history of a nonspecific diffuse transient erythematous rash in the neonatal period and a sibling death in the neonatal period attributed to meconium aspiration syndrome. On examination, the child was pale, dyspneic, and wasted, weighing $7.5 \mathrm{~kg}$ with multiple healed scars and clubbing. We also noticed a prominent forehead with a fleshy nasal tip. Respiratory examination revealed bilateral coarse crepitations, more on the right.

Initial investigations showed $\mathrm{Hb}-8.8 \mathrm{~g} / \mathrm{dL}, \mathrm{TC}-32,890 / \mathrm{mm}^{3}$ ( $\mathrm{N}-74.5 \%, \mathrm{~L}-22 \%, \mathrm{E}-0.6 \%)$. Chest $\mathrm{X}$-ray showed right upper and mid-zone opacity with bilateral infiltrates (Fig. 1). High-resolution CT chest revealed mediastinal lymphadenopathy with right-sided nodular infiltrates and bilateral bronchiectasis (Fig. 2).

The AFB stain and mycobacterial PCR by CBNAAT were repeated on gastric aspirate sample and found to be negative. The bronchoalveolar lavage (BAL) was again negative for TB but grew 


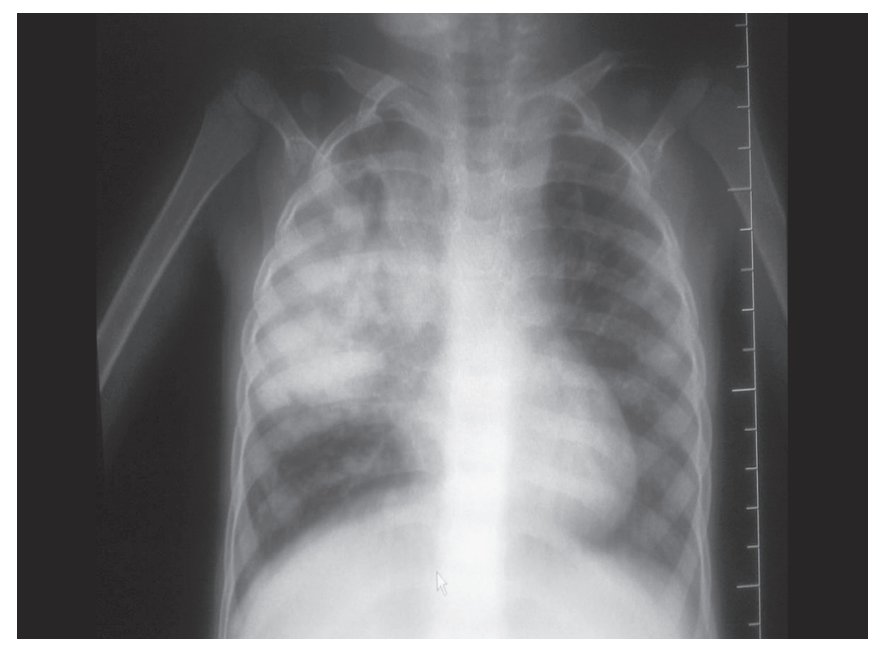

Fig. 1: Chest X-ray at presentation showing right upper and mid-zone opacities

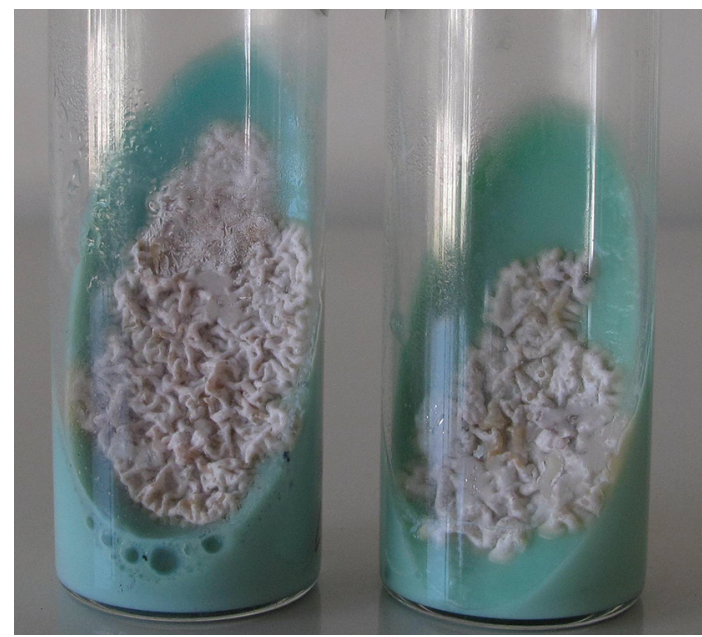

Fig. 3: Growth of Nocardia species in culture on Lowenstein-Jensen (LJ) medium

Aspergillus fumigatus. The positive serum galactomannan assay confirmed invasive aspergillosis. Meanwhile, the earlier mediastinal lymph node aspirate done elsewhere, cultured on the Lowenstein Jensen (LJ) medium grew Nocardia species (Fig. 3).

Immunodeficiency evaluation showed negative retrovirus status, normal neutrophil function, and normal lymphocyte subset assay. Serum immunoglobulin estimation revealed lgA-137 mg/ dL (27-195 mg/dL), lgG—2107 mg/dL (504-1464 mg/dL), lgM-202 $\mathrm{mg} / \mathrm{dL}$ (24-210 mg/dL), and $\mathrm{lgE}>2500 \mathrm{IU} / \mathrm{mL}(100-200 \mathrm{lU} / \mathrm{mL})$.

Based on the National Institute of Health-Hyper Immunoglobulin E syndrome (NIH-HIES) scoring system, our child had a score of 45, which is $75 \%$ of the maximal score in the 3-6 year age group, suggesting a diagnosis of possible HIES. ${ }^{2}$ Also, our child had a score of 34 on a recent scoring system suggested by Woellner et al. to diagnose HIES with STAT 3 mutations ${ }^{3}$ (Table 1). Based on the clinical scenario, autosomal dominant HIES was likely. However, due to financial constraints, we could not test for Th17 deficiency and STAT 3 mutation and a diagnosis of possible HIES was made. Considering the respiratory distress and clinical, microbiological, as well as radiological features ascribable to either invasive aspergillosis or pulmonary nocardiosis, we decided to treat both

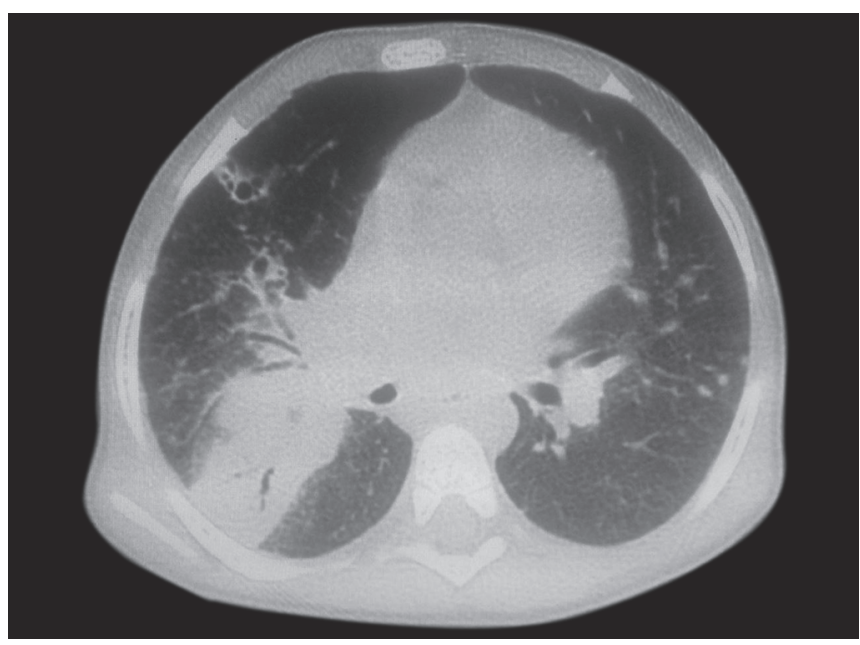

Fig. 2: HRCT chest showing right upper lobe consolidation, mediastinal lymphadenopathy, and bilateral bronchiectasis

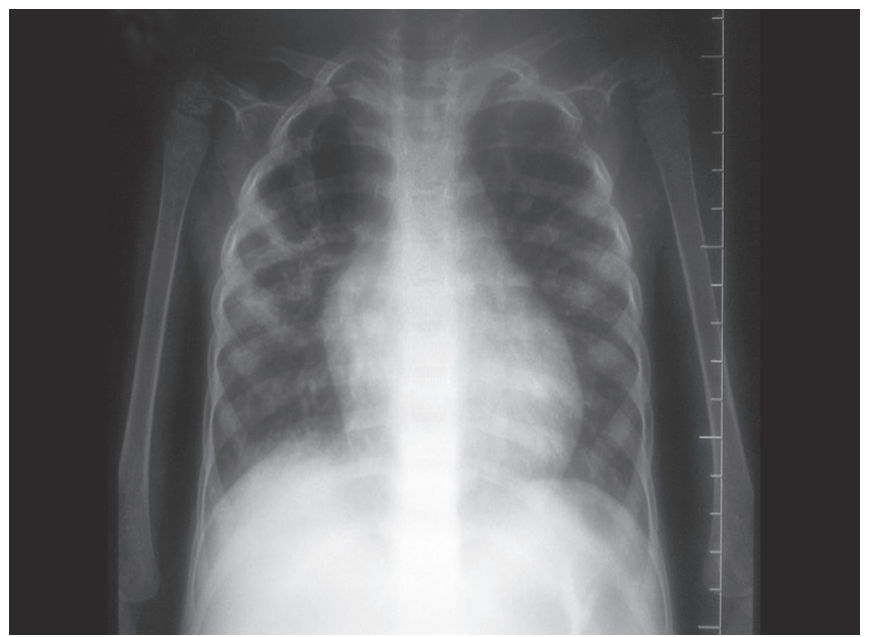

Fig. 4: Chest X-ray after 3 months of treatment showing radiological improvement

Table 1: Basis of diagnosis of HIES in our child based on established scoring systems

\begin{tabular}{lcl}
\hline Clinical characteristic of index case & $\begin{array}{l}\text { NIH HIES } \\
\text { scoring }\end{array}$ & $\begin{array}{l}\text { Scaled scoring- } \\
\text { Woellner et al. }\end{array}$ \\
\hline Highest IgE $\geq 2000 \mathrm{IU} / \mathrm{mL}$ & 10 & \\
Skin abscesses $\geq 4$ & 8 & \\
X-ray proven pneumonias $\geq 3$ & 8 & $8 \times 2.5$ \\
Parenchymal lung abnormality- & 6 & \\
bronchiectasis & & \\
Other serious infections-severe & 4 & \\
Newborn rash & 4 & $4 \times 2.08$ \\
Characteristic face-mild & 2 & $2 \times 3.33$ \\
Young age add on-2-5 years & 3 & \\
Total & 45 & 34 \\
\hline
\end{tabular}

with voriconazole and cotrimoxazole, respectively. The child improved symptomatically with resolution of fever and dyspnea, weight gain, and significant radiological improvement was noted at 3 months' follow-up (Fig. 4). We had planned to continue treatment for a year. Parents were also counseled for stem cell transplantation 
after genetic confirmation. Unfortunately, the child was lost to follow-up after 6 months of treatment.

\section{Discussion}

The HIESs comprise a group of primary immunodeficiency disorders that exhibit markedly elevated IgE levels, recurrent staphylococcal skin abscesses, eczema, and pulmonary infections. ${ }^{2}$ Similarly, our child presented with multiple skin abscesses and pulmonary infection. Both autosomal dominant and autosomal recessive forms of the disorder have been described. Most autosomal dominant HIESs (AD-HIESs) have been found to be due to mutations in STAT3. The skin and lung infections in AD-HIES might be a result of deficient Th17 differentiation. ${ }^{2}$ Internal abscesses like testicular abscess, seen in our child, have been reported in HIES with STAT3 mutations. ${ }^{4}$ Recurrent and severe pulmonary infections are the hallmark of HIES. Aberrant healing following these infections leads to pneumatoceles and bronchiectasis seen in $75 \%$ of the patients. ${ }^{2}$ These heterogeneous manifestations are often misdiagnosed as tuberculosis in endemic tropical countries. ${ }^{5}$ There are many case series from India for HIES wherein atypical features have been highlighted; ${ }^{6}$ however, this coinfection pattern is unique and not reported.

The most common causative organism is Staphylococcus aureus. However, once there is parenchymal lung disease, infections with fungi, nontuberculous mycobacteria, and Pseudomonas are known to occur. Growth of Aspergillus fumigatus from BAL fluid, along with positive serum galactomannan assay, suggested invasive aspergillosis in our child. Vinh et al. described invasive fungal infections, most commonly Aspergillus typically occurring in AD-HIES patients with concurrent anatomical lung defects from previous bacterial pneumonias. ${ }^{7}$ However, invasive fungal disease typically occurred in the fourth decade of life and only when there were concurrent anatomical lung defects from previous bacterial pneumonias. ${ }^{7}$ Invasive aspergillosis at such an early age has rarely been reported earlier. ${ }^{8}$

Nocardiosis is a rare opportunistic disease caused by the actinomycete Nocardia spp., mainly affecting the immunocompromised. Pulmonary nocardiosis is the commonest manifestation seen more often in patients with structural pulmonary disease. ${ }^{9}$ Clinical presentations include acute, subacute, and chronic pneumonia with radiographic evidence of lobar infiltrates, abscesses, cavities, pleural effusion, or pulmonary nodules, making tuberculosis a close differential. ${ }^{9}$ The index child had radiological findings of pulmonary nodules. Direct smears from Nocardia show gram-positive, beaded, branching acid fast filaments. These acid-fast filaments can be easily confused with TB bacilli. Mycobacteria do not stain well with Gram stain and modified acid-fast stain and hence can be differentiated. ${ }^{10}$ In the index case, lack of clinical response to ATT, presence of pulmonary nodules, and gram-positive AFB on smear raised the suspicion of nocardiosis. Culture on the LJ medium grew Nocardia. Nocardia on nonselective culture media grow as colonies with chalky white or cotton-ball appearance because of the presence of abundant aerial filaments, which again differentiate them from mycobacteria. On these grounds, a diagnosis of nocardiosis was made and the child was started on co-trimoxazole.

Nocardiosis occurs infrequently in children. Lee et al. reported the first case of pulmonary nocardiosis in a 10-month-old child with HIES in 1999.' The child with recurrent sinopulmonary bacterial infections, numerous cold staphylococcal abscesses, severe extensive eczematoid rash, and markedly elevated IgE levels presented with a mediastinal mass that showed Nocardia on ZN stain, similar to our case. The child was also started on co-trimoxazole but developed Steven Johnson syndrome and succumbed to it.

The literature review revealed a few case reports of nocardiosis and aspergillosis coinfections mostly in the immunocompromised adults $^{11,12}$ and in patients with structural lung diseases ${ }^{13}$ but none in children.

\section{Conclusion}

As we diagnose more and more children with primary immunodeficiencies, it is important to look for rare opportunistic as well as coinfections in them. To the best of our knowledge, ours is the first report of invasive pulmonary aspergillosis and nocardiosis in a child with HIES.

\section{Clinical Significance}

This emphasizes the importance of looking for opportunistic as well as coinfections in children with primary immunodeficiencies.

Albeit much rarer, aspergillosis and pulmonary nocardiosis can be close mimicks of tuberculosis. Nonresolving pulmonary symptoms in the immunocompromised host must raise a suspicion of opportunistic infections including nocardiosis as early diagnosis and treatment may alter outcomes.

\section{References}

1. Lee WS, Boey CC, Goh AY. Pulmonary nocardiosis in a child with hyperimmunoglobulin E syndrome. Singapore Med J 1999;40:280.

2. Grimbacher, et al. Diagnosing the hyper IgE syndrome: incidence of clinical features. Inmunologia 2002;21:2-4.

3. Woellner C, Gertz EM, Schäffer AA. Mutations in STAT3 and diagnostic guidelines for hyper-IgE syndrome. J Allergy Clin Immunol 2010;125(2):424-432. DOI: 10.1016/j.jaci.2009.10.059.

4. Schimke LF, Sawalle-belohradsky J, Roesler J, et al. Diagnostic approach to the hyper-IgE syndromes: immunologic and clinical key findings to differentiate hyper-IgE syndromes from atopic dermatitis. J Allergy Clin Immunol 2010;126(3):611-7.e1. DOI: 10.1016/j. jaci.2010.06.029.

5. Singh A, Mandal A, Seth R. Hyper IgE syndrome: often a missed diagnosis. Int J Contemp Pediatr 2016;3:674-677. DOI: 10.18203/23493291.ijcp20161064.

6. Patel NH, Padhiyar JK, Shah YB, et al. Unusual presentations and associations of hyper IgE syndrome: retrospective analysis of ten cases at tertiary care institute - with review of indian published reports. Indian J Paediatr Dermatol 2018;19(1):31-36. DOI: 10.4103/ijpd.IJPD_144_16.

7. Vinh DC, Sugui JA, Hsu AP, et al. Invasive fungal disease in autosomal-dominant hyper-IgE syndrome. J Allergy Clin Immunol 2010;125(6):1389-1390. DOI: 10.1016/j.jaci.2010.01.047.

8. Fan $H$, Huang $L$, Yang $D$, et al. Pediatric hyperimmunoglobulin $E$ syndrome: a case series of 4 children in China. Medicine (Baltimore) 2018;97(14):e0215. DOI: 10.1097/MD.0000000000010215.

9. Ambrosioni J, Lew D, Garbino J. Nocardiosis: update clinical review and experience at a tertiary center. Infection 2010;38(2):89-97. DOI: 10.1007/s15010-009-9193-9.

10. Minero MV, Marín M, Cercenado $E$, et al. Nocardiosis at the turn of the century. Medicine 2009;88(4):250-261. DOI: 10.1097/ MD.0b013e3181afa1c8.

11. Hamadani M, Benson Jr DM, Blum W, et al. Nocardia and aspergillus co-infection in a patient with chronic graft-versus-host disease. Transpl Infect Dis 2008;10(1):24-26. DOI: 10.1111/j.1399-3062.2007.00268.x.

12. Uppal G, Potula R, Truant AL. Nocardia and aspergillus pulmonary infection in a patient on long-term steroid treatment: case report and literature review. Clin Microbiol Newslett 2015;37(5):35-39. DOI: 10.1016/j.clinmicnews.2015.02.001.

13. Meena DS, Kumar D, Bohra GK, et al. Pulmonary nocardiosis with aspergillosis in a patient with COPD: a rare co-infection. IDCases 2020;20:e00766. DOI: 10.1016/j.idcr.2020.e00766. 